\title{
Genetics plays a role in nevi distribution in women
}

\author{
Alessia Visconti*,1 (D), Marianna Sanna1, Veronique Bataille ${ }^{1,2}$ \& Mario Falchi ${ }^{1}$ \\ ${ }^{1}$ Department of Twin Research \& Genetic Epidemiology, King's College London, London, UK \\ ${ }^{2}$ Department of Dermatology, West Herts NHS Trust, Herts, UK \\ *Author for correspondence: alessia.visconti@kcl.ac.uk \\ " ${ }^{\text {it }}$ would be crucial to translate these results to melanoma research, investigating whether these \\ pleiotropic loci, as well as any other associated locus, are exerting an effect on melanoma \\ susceptibility that is sex dependent."
}

First draft submitted: 29 November 2019; Accepted for publication: 20 December 2019; Published online: 17 March 2020

Keywords: melanoma • nevi • sex-associated differences

Melanoma is a tumor whose incidence has increased steadily in the last 50 years in fair-skinned populations. Despite representing less than $5 \%$ of all skin cancers, melanoma accounts for the majority of skin cancer deaths [1].

Men have a poorer prognosis compared with women and, in many countries, melanoma incidence is higher in women [2,3]. These sex differences could be due to both behavioral and biological factors, as reviewed by Schwartz et al. [3]. Behavioral factors influenced by gender may include, among others, sunbed use, recreational UV exposure, use of sun protection and skin awareness. The increased risk in women appears to be counterbalanced by increased protection and early diagnosis, which allows for the detection of thinner tumors, improving the survival rate. However, biological factors, such as differences in the underlying genetic risk architecture, can also be at play [3]. Males and females also present different primary tumor sites, with a higher incidence on the head, neck and torso in males and on the legs in females. It is not yet clear whether this could be due to sex differences in behaviors, genetic make-up, or their interplay [3].

Melanoma and nevi both derive from the proliferation of melanocytes, which is uncontrolled in the former and benign in the latter. It is estimated that between 20 and 50\% of melanomas arise from a nevus [4]. The total body nevus count is the most powerful phenotypic marker to predict melanoma risk [5], and melanoma and nevi share multiple genetic and environmental influences [6]. For instance, studies suggest that early exposure to UV light plays a role in inducing both nevi and melanoma (e.g., [7]), and a recent genome-wide meta-analysis by Duffy et al. identified pleiotropic effects on melanoma risk of five genetic loci (namely: IRF4, DOCK8, MTAP, 9q31.2, KITLG and PLA2G6) influencing nevus count [8].

Mirroring what is already observed for melanoma primary site, sex differences in nevus distribution are observed at different body sites from childhood onward, with females having more nevi on the legs, and males having more nevi on the head, neck and torso [9,10]. Interestingly, nevus count on the torso in males and on the legs in females are better predictors of overall melanoma risk than nevus count at any other site [9]. These sex differences in nevus counts could be due to both behavioral and genetic factors, with observational studies supporting both hypotheses. For instance, a small study of men and women living in Spain failed to find any difference in nevus count on the legs, likely due, according to the authors, to the fact that, in this country, both men and women commonly expose this site [11]. However, a much larger study carried out in Queensland, Australia, where again children of both sexes commonly expose their legs, observed an increased number of nevi on the legs in girls compared with boys [12]. Furthermore, sex chromosomes may also play a role in nevus distribution and number. For instance, individuals with Turner syndrome (45,X monosomy) show an increased number of nevi [13], and a study exploring genes differentially expressed in Turner syndrome versus healthy women (46,XX) identified, among the others, an autosomal gene (DOCK 7 ) associated to the distribution and function of melanocytes in mice [14]. Another hypothesis is that the observed differences in body site nevus distribution between sexes could

Future $\because$ Medicine 
be due to sex-specific mechanisms driving melanocyte migration and differentiation during embryogenesis, and which are likely to continue affecting nevi and melanoma behavior throughout one's lifetime, an aspect also worth investigating.

To investigate the role of genetic and environmental factors in these site-specific differences in women, we took advantage of nevus count data available for 1512 female twin pairs of European ancestry. Twins studies can estimate the proportion of the variability of a trait that is caused by genetic effect and by environmental factors [15]. We showed that $69 \%$ of the nevus count variability on the legs - the site more common for melanoma in women could be explained by genetic effects, and only $31 \%$ was due to environmental factors [10]. For comparison, on the torso, where melanoma incidence is lower in females, genetic effects were the lowest and responsible for only $26 \%$ of the nevus count variability in the female twins. In the same group of female twins, genetics explained $34 \%$ of the nevus count variability on the head and neck, and $54 \%$ in the arms.

Next, we investigated whether genetic variants at the six pleiotropic melanoma and nevus count loci identified by Duffy et al. [8] had a different effect on nevus count distribution at different body sites in 2864 women. We observed that genetic variants at the IRF4,9q31.2, and PLA2G6 loci influenced the number of nevi on the legs. Nevus count on the arms was significantly associated with variants at the DOCK8, MTAP and PLA2G6 loci, while nevus count on the head and neck were associated with variants at the IRF4 and MTAP loci. Only the PLA2G6 locus was associated with nevus count distribution on the torso [10].

Our results suggest that, in women, increased nevus count on the legs is under significant genetic control, and unlikely due to higher UV exposure alone, as already suggested [16], and that the impact of genetics on nevus count differs according to body sites.

In summary, current research demonstrates that melanoma susceptibility and survival are strongly connected with biological sex, which appears to influence also the site of the primary tumor [3]. Analogously, nevus count distribution at different body sites seems to have a sex-specific pattern, which we have shown to be under genetic influence [10], albeit only in women.

In the future, it would be important, first and foremost, to assess whether nevus count distribution is also under a site-specific genetic control in men, which we could not test due to the small number of men in our cohort, and to extend our investigation to the role of sex chromosomes. Then, it would be crucial to translate these results to melanoma research, investigating whether these pleiotropic loci, as well as any other associated locus, are exerting an effect on melanoma susceptibility that is sex dependent. Indeed, few studies have investigated genetic differences according to sex in melanoma (reviewed in [3]). This would include performing sex-stratified genome-wide association studies or candidate gene analyses, as well as testing for gene-by-sex interactions. Of course, this sex-stratified approach of investigation could be extended to other melanoma risk factors, such as hair and eye color, or tanning ability. In our view, these approaches will allow dissection of the underlying mechanisms responsible for the observed differences in melanoma susceptibility between sexes, and drive intervention strategies targeted to men and women distinctly to improve therapeutic outcomes.

\section{Author contributions}

A Visconti wrote the manuscript. M Sanna contributed to the literature review. All authors read and approved the final version of the manuscript.

\section{Financial \& competing interests disclosure}

M Falchi has received research funding from Sanofi. The authors have no other relevant affiliations or financial involvement with any organization or entity with a financial interest in or financial conflict with the subject matter or materials discussed in the manuscript apart from those disclosed.

No writing assistance was utilized in the production of this manuscript.

\section{Open access}

This work is licensed under the Attribution-NonCommercial-NoDerivatives 4.0 Unported License. To view a copy of this license, visit http://creativecommons.org/licenses/by-nc-nd/4.0/ 


\section{References}

Papers of special note have been highlighted as: $\bullet$ of interest $\bullet \bullet$ of considerable interest

1. Matthews NH, Li WQ, Qureshi AA et al. Chapter 1: Epidemiology of melanoma. In: Cutaneous Melanoma: Etiology and Therapy [Internet]. Ward WH, Farma JM (Eds). Codon Publications, Brisbane, AU (2017).

2. Joosse A, de Vries E, Eckel R et al. Gender differences in melanoma survival: female patients have a decreased risk of metastasis. J. Invest. Dermatol. 131(3), 719-726 (2011).

3. Schwartz MR, Luo L, Berwick M. Sex differences in melanoma. Curr. Epidemiol. Rep. 6(2), 112-118 (2019).

-• Review of sex differences in melanoma incidence and survival.

4. Shitara D, Nascimento MM, Puig S et al. Nevus-associated melanomas: clinicopathologic features. Am. J. Clin. Pathol. 142(2), 485-491 (2014).

5. Bhatt M, Nabatian A, Kriegel D et al. Does an increased number of moles correlate to a higher risk of melanoma? Melanoma Manag. 3(2), 85-87 (2016).

6. Whiteman DC, Watt P, Purdie DM et al. Melanocytic nevi, solar keratoses, and divergent pathways to cutaneous melanoma. J. Natl Cancer Inst. 95(11), 806-812 (2003).

7. Augustsson A. Nevi and initiation by sun. In: Skin Cancer and UV Radiation. Altmeyer P, Hoffmann K, Stücker M. (Eds). Springer Berlin Heidelberg, Heidelberg, Germany, 589-594 (1997).

8. Duffy DL, Zhu G, Li X et al. Novel pleiotropic risk loci for melanoma and nevus density implicate multiple biological pathways. Nat. Commun. 9(1), 4774 (2018).

9. Krüger S, Garbe C, Büttner P et al. Epidemiologic evidence for the role of melanocytic nevi as risk markers and direct precursors of cutaneous malignant melanoma. J. Am. Acad. Dermatol. 26(6), 920-926 (1992).

10. Visconti A, Ribero S, Sanna M et al. Body site-specific genetic effects influence naevus count distribution in women. Pigment Cell Melanoma Res. doi:10.1111/pcmr.12820 (2019) (Epub ahead of print).

- The first study investigating body site-specific genetic effects influencing the development of nevi in women.

11. Echeverría B, Bulliard J-L, Guillén C et al. Indicators for the total number of melanocytic naevi: an adjunct for screening campaigns. Observational study on 292 patients. Br. J. Dermatol. 170, 144-149 (2014).

12. MacLennan R, Kelly JW, Rivers JK et al. The Eastern Australian Childhood Nevus Study: site differences in density and size of melanocytic nevi in relation to latitude and phenotype. J. Am. Acad. Dermatol. 48(3), 367-375 (2003).

13. Becker B, Jospe N, Goldsmith LA. Melanocytic nevi in Turner syndrome. Pediatr. Dermatol. 11(2), 120-124 (1994).

14. Trolle C, Nielsen MM, Skakkebæk A et al. Widespread DNA hypomethylation and differential gene expression in Turner syndrome. Sci. Rep. 6, 34220 (2016).

15. Verweij KJH, Mosing MA, Zietsch BP et al. Estimating heritability from twin studies. In: Statistical Human Genetics. Elston RC, Satagopan JM, Sun S. (Eds). Humana Press, NJ, USA, 151-170 (2012).

16. Bataille V. Melanoma. Shall we move away from the sun and focus more on embryogenesis, body weight and longevity? Med. Hypotheses 81(5), 846-850 (2013). 
